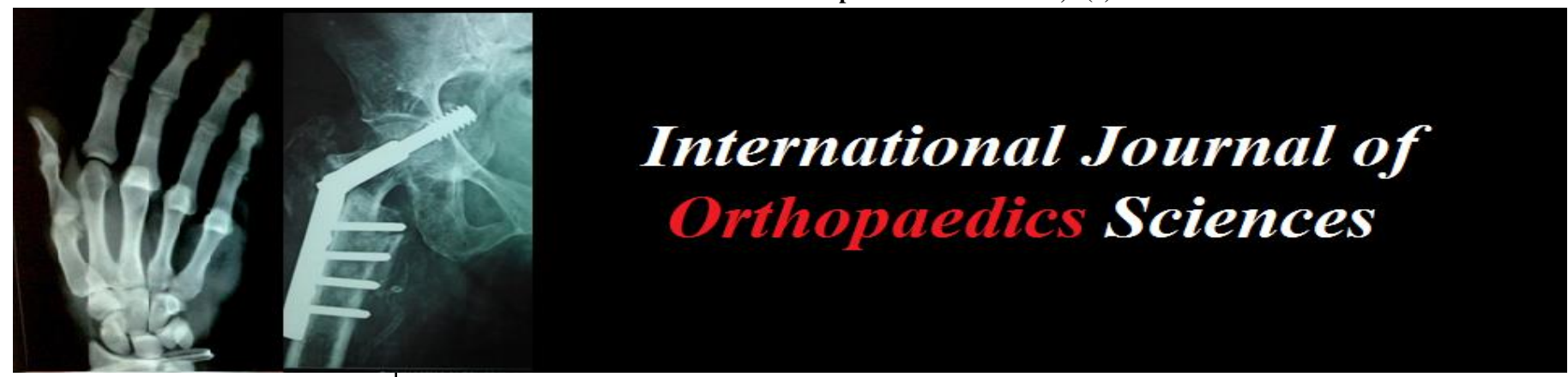

E-ISSN: 2395-1958

P-ISSN: 2706-6630

IJOS 2021; 7(3): 470-474

(C) 2021 IJOS

www.orthopaper.com

Received: 17-05-2021

Accepted: 21-06-2021

Dr. Pramod Singh Chahar Resident Doctor, SMS Medical

College, Jaipur, Rajasthan, India

Dr. Ravinder Kumar Lamoria

Associate Professor, SMS

Medical College, Jaipur

Dr. Amit Kumar Gupta

Resident Doctor, SMS Medical

College, Jaipur, Rajasthan, India

Dr. Gyanendra Singh Chauhan Senior Resident, RNT Medical College, Udaipur, Rajasthan, India

Dr. Mahesh Chand Bansal Sr. Professor and Unit Head, SMS Medical College, Jaipur, Rajasthan, India
Corresponding Author: Dr. Pramod Singh Chahar Resident Doctor, SMS Medical College, Jaipur, Rajasthan, India

\section{Prospective comparative study of early outcomes of fixed closed loop and adjustable loop femoral fixation techniques used for arthroscopic anterior cruciate ligament reconstruction}

\author{
Dr. Pramod Singh Chahar, Dr. Ravinder Kumar Lamoria, Dr. Amit \\ Kumar Gupta, Dr. Gyanendra Singh Chauhan and Dr. Mahesh Chand \\ Bansal
}

DOI: https://doi.org/10.22271/ortho.2021.v7.i3g.2791

\section{Abstract}

Background: Depending on the severity of your ACL injury, treatment may include rest and rehabilitation exercises to help you regain strength and stability or surgery to replace the torn ligament followed by rehabilitation. Methods of femoral fixation in arthroscopic ACL reconstruction surgery are widely discussed in the literature. The cortical suspension technique is a popular option with a variety of adjustable loop and fixed closed loop devices available in the market.

Aim: To evaluate short term outcome of arthroscopic ACL reconstruction using fixed closed loop fixation (CLF) and adjustable loop fixation (ALF) techniques.

Study Design: Hospital based Prospective randomized comparative interventional study.

Materials and Methods: 60 cases were included in this study, in which 30 cases were treated arthroscopically by using CLF and remaining 30 cases were operated arthroscopically by using ALF. Clinical outcome was assessed using Lachman and pivot shift, and results were graded using the International Knee Documentation Committee. Functional outcome was evaluated by using Lysholm score up to 8 months.

Result: Lachman grading (mm) pre-operative in CLF group was $2.84 \pm 0.35 \mathrm{~mm}$ and in ALF group was 2.72 \pm 0.31 . Maximum patients were from IKDC 2+ grade. $70.00 \%$ in CLF group and $56.67 \%$ in ALF group. Lysholm score pre-operative in CLF group was 63.00 \pm 1.29 and in ALF group was $62.90 \pm 1.86$. Lachman grading $(\mathrm{mm})$ post-operative in CLF group was $0.31 \pm 0.02 \mathrm{~mm}$ and in ALF group was $0.35 \pm 0$. $06 \mathrm{~mm}$.post-operative $16.67 \%$ patients belong IKDC $2+$ grade in CLF group and in $10.00 \%$ patients in ALF group. Lysholm score post-operative in CLF group was 92.36 \pm 0.95 and in ALF group was $91.9 \pm$ 0.91There were no statistically significant differences.

Conclusion: We concluded that in femoral fixation of ACL graft both CLF and ALF techniques may provide secure fixation, equal reduction of graft laxity, and similar functional outcome in ACL deficient knee.

Keywords: adjustable loop fixation, anterior cruciate ligament reconstruction, closed loop fixation, cortical suspensory fixation devices

\section{Introduction}

The knee is one of the most commonly injured joint. Knee injury is the second most common problem in the musculoskeletal system for which patients consult their physician [1]. An anterior cruciate ligament (ACL) injury occurs when the ACL is either stretched, partially torn, or completely torn. The most common injury is a complete tear. It is more common in athletes, particularly those who participate in alpine skiing, football (soccer), American football, or basketball ${ }^{[2]}$. Depending on the severity of your ACL injury, treatment may include rest and rehabilitation exercises to help you regain strength and stability or surgery to replace the torn ligament followed by rehabilitation ${ }^{[3]}$. The anterior cruciate ligament is one of the four main ligaments of the knee, providing $85 \%$ of the restraining force to anterior tibial displacement at 30 degrees and 90 degrees of knee flexion. The ACL is the most injured ligament of the four located in the knee ${ }^{[4]}$. 
There are two bundles of the ACL: the anteromedial and the posterolateral, named according to where the bundles insert into the tibial plateau. The ACL attaches in front of the intercondyloid eminence of the tibia, where it blends with the anterior horn of the medial meniscus ${ }^{[5]}$. Arthroscopic ACL reconstruction has remained the gold standard for ACL injuries, particularly in young individuals, sportsman and athletes who is aiming to return his or her pre injury level of activity [6]. Methods of femoral fixation in ACL reconstruction surgery are widely discussed in the literature. The cortical suspension technique is a popular option with a variety of adjustable loop and fixed closed loop devices available in the market. Femoral cortical suspension devices have gained popularity because of the simplicity, reliability, and excellent tensile strength ${ }^{[7]}$. Techniques have been described that allow direct arthroscopic visualization of the fixation button during application in the femur, helping to avoid some of the commonly cited complications. We evaluated short-term outcome (considered as $8^{\text {th }}$ months) following arthroscopic ACL reconstruction by using CLF and ALF device with semitendinosus and gracilis tendon autograft.

\section{Materials and Methods \\ Study Area}

Department of orthopedics in S.M.S medical college and attached group of hospitals Jaipur, Rajasthan.

\section{Study Design}

Hospital based Prospective Randomized comparative interventional study.

\section{Study Duration}

Data collection was started first after the approval from Institutional Research Review Board and Ethical Committee up to June 2020 or till sample size is achieved, whichever is earlier. Then it will take one month for data processing and writing thesis.

\section{Sample Size}

Sample size was calculated 28 subjects of each of these two groups at error 0.05 and power $80 \%$ assuming difference of means in Lysholm score (Post-Operative to Pre-Operative) of CLF and ALF 4.0 with SD 5.39 as per seed article. So, for study purpose, 30 cases for ACL Reconstruction with CLF Technique and 30 cases for ACL Reconstruction with ALF Technique was taken by simple random selection.

\section{Inclusion Criteria}

1. Diagnosed to have complete ACL tear clinically and radiologically on magnetic resonance imaging (MRI).

2. Age group 18 years and above.

3. Patent who will give written informed consent to take Part in study

4. Patients who are fit for anaesthesia and surgery.

\section{Exclusion Criteria}

1. Pregnancy

2. Uncontrolled Diabetes Mellitus, Hypertension, Psychiatric illness, Acute Myocardial Infarction.

3. Previous knee surgery

4. Revision ACL

5. Bilateral ACL rupture

6. Other musculo-skeleton involvement in the same limb

7. Collateral ligaments and PCL injury
Surgery was done under spinal or epidural anaesthesia or both. A high pneumatic tourniquet was used in all cases. We established an anterolateral and an anteromedial portal. The longitudinal incision about $3-4 \mathrm{~cm}$ was given medial to the tibial tubercle. The gracilis and the semitendinosus tendons are identified lying beneath the sartorial fascia. Then graft is harvested by using tendon stripper.

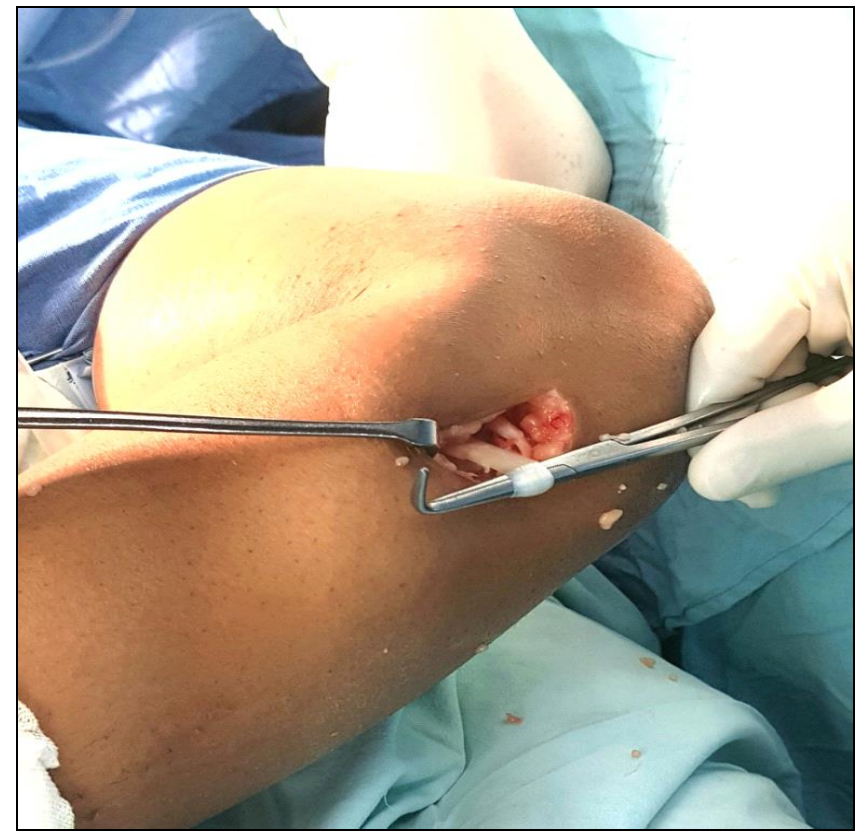

Fig 1: Graft harvesting

Usually, the two tendon lengths which are obtained in $85 \%$ of cases in our study that is semitendinosus (average $285 \mathrm{~mm}$ ) and gracilis (average $190 \mathrm{~mm}$ ) is doubled on itself. Henceforth both the ends of tendon are sutured i.e., whipstitch sutures and then both the grafts are placed side to side and doubled on itself, so that a quadruple graft is prepared. The graft diameter is now measured.

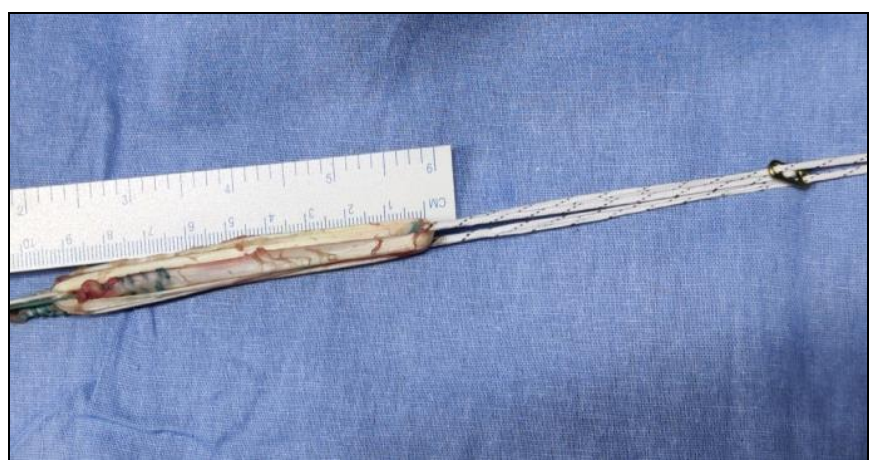

Fig 2: Graft loaded on ALF device

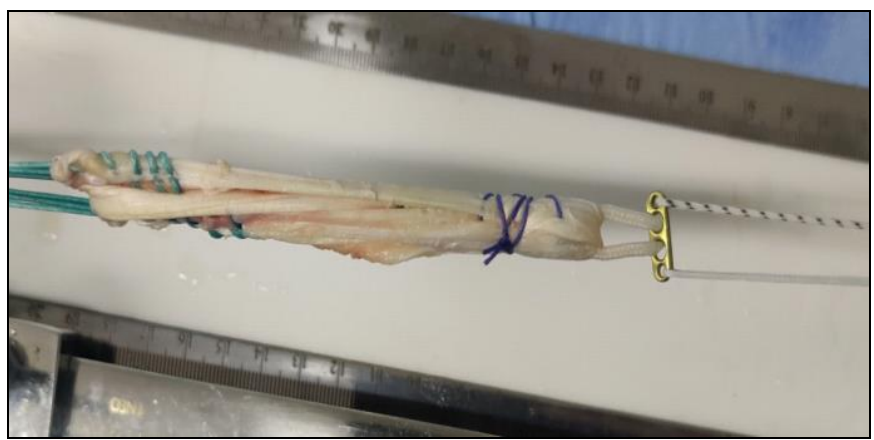

Fig 3: Graft loaded on CLF device 


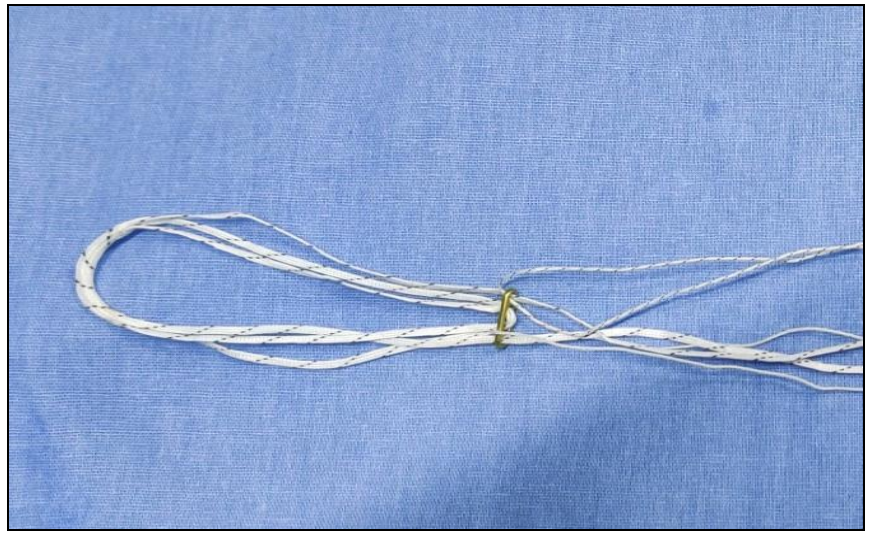

Fig 4: Adjustable loop device for femoral fixation

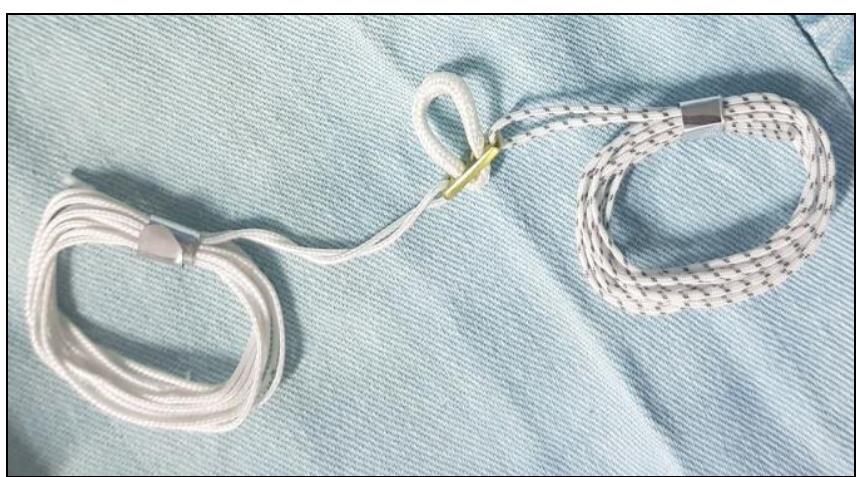

Fig 5: Fixed close loop device for femoral fixation

Remnants of the torn ACL are resected. An attempt is made to preserve some of the ACL fibers at the tibial, insertion site to aid with placement of the tibial tunnel. We aim to place the femoral tunnel in the center of the ACL femoral insertion site at 10:00/ 2:00 "0 clock position for right / left knee respectively. A $2.7 \mathrm{~mm}$ guide pin is inserted through appropriate size of offset aimer and tapped into the bone until the drill-tip part of the pin is flush with the cortex. The femoral tunnel is drilled through the femoral cortex using a $4.5 \mathrm{~mm}$ Endobutton drillbit. The length of the femoral tunnel can be determined by noting the length on the drillbit at the time of cortical breakthrough. Knowing the length of the femoral tunnel allows the femoral socket to be drilled to the femoral cortex. Drilling the femoral socket to the cortex will allow room for the CLF/ALF device (endobutton) to flip in all situations, eliminating the need to calculate how deep to drill the femoral socket. The ACL tibial aimer is typically set at 55 degrees and the tunnel started $1 \mathrm{~cm}$ medial to the crest of the tibia. The tip of the tibial guide pin should be positioned 2-3 $\mathrm{mm}$, anterior to the anterior horn of the lateral meniscus and $7 \mathrm{~mm}$ anterior to PCL, and2-3 $\mathrm{mm}$ anterior to peak of medial spine. Ideally, the tibial guide pin should be placed in the medial half of ACL tibial insertion site. Once proper placement of tibial guide pin is confirmed, the tibial tunnel is drilled in the conventional fashion using a fully fluted cannulated drill bit. The quadrupled hamstring graft is then loaded on CLF/ALF device (endobutton). A pin with a long suture attached to the eyelet is passed through the accessory AM portal and out through the femoral tunnel. The looped suture is visualized in the joint and retrieved with an arthroscopic suture grasper through the tibial tunnel. The graft is passed through this suture and threads of CLF/ALF device (endobutton) are taken out through femoral tunnel. The green threads of CLF/ALF device (endobutton) are pulled from the femoral tunnel side and passage of graft is visualized with the arthroscope. The flipping of CLF/ALF device as it crosses the lateral femoral cortex is felt and confirmed by flipping the two threads or by a C-arm. The cycling motion of knee is done 15-20 times with knee in 20-30 degrees of flexion. Tibial tunnel is fixed with a bioscrew of appropriate size depending on the graft diameter and length of tibial tunnel.

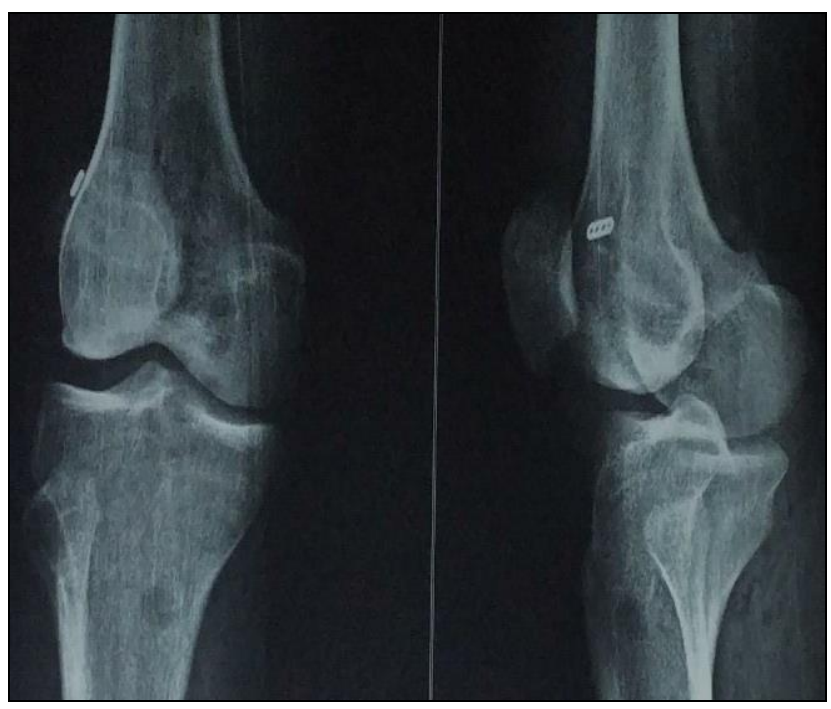

Fig 6: Post-operative x-ray

Postoperative rehabilitation was similar for both groups. Full weight bearing ambulation and knee range of motion exercises were started within 48 hours after surgery. No rapid recovery protocol or continuous passive motion was applied to any of the patients. Patients were discharged home after 7 days, postoperatively. Patients were followed up regularly in the outpatient department at every 2 weeks till a total of 12 weeks, every month till next 2 months and every 3 months thereafter. Evaluation in the follow up period is carried out through Lysholm score, which is a subjective score, patient's range of motion and post-op stability is assessed by Lachman test and Pivot shift test.

\section{Observation and Results}

Table 1: Age wise distribution of study subjects

\begin{tabular}{|c|c|c|c|}
\hline Age in Yrs & CLF & ALF & P-value \\
\hline Mean & 26.92 & 26.90 & 0.512 \\
\hline SD & 3.12 & 3.18 & \\
\hline
\end{tabular}

In present study, mean age in CLF group was 26.92 \pm 3.12 Yrs and in ALF group was $26.90 \pm 3.18$ Yrs. Both groups were comparable

Table 2: Sex wise distribution of study subjects

\begin{tabular}{|c|c|c|c|c|c|}
\hline \multirow{2}{*}{ Sex } & \multicolumn{2}{|c|}{ CLF } & \multicolumn{2}{|c|}{ ALF } & \multirow{2}{*}{ P-value } \\
\cline { 2 - 5 } & No & \% & No & \% & \\
\hline Male & 28 & 93.33 & 28 & 93.33 & 0.512 \\
\hline Female & 2 & 6.67 & 2 & 6.67 & \\
\hline Total & 30 & 100.00 & 30 & 100.00 & \\
\hline
\end{tabular}

In present study, Male patients $(93.33 \%)$ were more than female patients $(6.67 \%)$. Both groups were comparable

Table 3: Lachman grading ( $\mathrm{mm}$ ) pre-operative wise distribution of study subjects

\begin{tabular}{|c|c|c|c|}
\hline Lachman grading (mm) pre-operative & CLF & ALF & P-value \\
\hline Mean & 2.84 & 2.72 & 0.234 \\
\hline SD & 0.35 & 0.31 & \\
\hline
\end{tabular}

In present study, Lachman grading (mm) pre-operative in CLF group was $2.84 \pm 0.35 \mathrm{~mm}$ and in ALF group was $2.72 \pm 0.31$ 
Table 4: Pivot shift grading pre-operative wise distribution of study subjects

\begin{tabular}{|c|c|c|c|c|c|}
\hline \multirow{2}{*}{$\begin{array}{l}\text { Pivot shift grading } \\
\text { pre-operative }\end{array}$} & \multicolumn{2}{|c|}{ CLF } & \multicolumn{2}{|c|}{ ALF } & \multirow{2}{*}{$\begin{array}{c}P \text { P- } \\
\text { value }\end{array}$} \\
\hline & No & $\%$ & No & $\%$ & \\
\hline IKDC $1+$ & 3 & 10.00 & 3 & 10.00 & 0.494 \\
\hline IKDC $2+$ & 21 & 70.00 & 17 & 56.67 & \\
\hline IKDC 3+ & 6 & 20.00 & 10 & 33.33 & \\
\hline Total & 30 & 100.00 & 30 & 100.00 & \\
\hline
\end{tabular}

In present study, Maximum patients were from IKDC 2+ grade $70.00 \%$ in CLF group and $56.67 \%$ in ALF group

Table 5: Lysholm score pre-operative wise distribution of study subjects

\begin{tabular}{|c|c|c|c|}
\hline Lysholm scorepre-operative & CLF & ALF & P-value \\
\hline Mean & 63.00 & 62.90 & 0.809 \\
\hline SD & 1.29 & 1.86 & \\
\hline
\end{tabular}

In present study, Lysholm score pre-operative in CLF group was $63.00 \pm 1.29$ and in ALF group was $62.90 \pm 1.86$

Table 6: Lachman grading ( $\mathrm{mm}$ ) post-operative wise distribution of study subjects

\begin{tabular}{|c|c|c|c|}
\hline $\begin{array}{c}\text { Lachman grading } \\
\text { (mm) post-operative }\end{array}$ & CLF & ALF & P-value \\
\hline Mean & 0.31 & 0.35 & 0.234 \\
\hline SD & 0.02 & 0.06 & \\
\hline
\end{tabular}

In present study, Lachman grading $(\mathrm{mm})$ post-operative in CLF group was $0.31 \pm 0.02 \mathrm{~mm}$ and in ALF group was $0.35 \pm 0.06 \mathrm{~mm}$

Table 7: Pivot shift grading post-operative wise distribution of study subjects

\begin{tabular}{|c|c|c|c|c|c|}
\hline \multirow{2}{*}{$\begin{array}{c}\text { Pivot shift grading } \\
\text { pre-operative }\end{array}$} & \multicolumn{2}{|c|}{ CLF } & \multicolumn{2}{|c|}{ ALF } & \multirow{2}{*}{$\begin{array}{c}P- \\
\text { value }\end{array}$} \\
\hline & No & $\%$ & No & $\%$ & \\
\hline IKDC 1+ & 0 & 0.00 & 0 & 0.00 & 0.494 \\
\hline IKDC 2+ & 5 & 16.67 & 3 & 10.00 & \\
\hline IKDC 3+ & 0 & 0.00 & 0 & 0.00 & \\
\hline
\end{tabular}

In present study, post-operative $16.67 \%$ patients belong IKDC $2+$ grade in CLF group and in $10.00 \%$ patients in ALF group

Table 8: Lysholm score post-operative wise distribution of study subjects

\begin{tabular}{|c|c|c|c|}
\hline Lysholm score post-operative & CLF & ALF & P-value \\
\hline Mean & 92.36 & 91.9 & 0.718 \\
\hline SD & 0.95 & 0.91 & \\
\hline
\end{tabular}

In present study, Lysholm score post-operative in CLF group was 92.36 \pm 0.95 and in ALF group was $91.9 \pm 0.91$

\section{Results}

Mean age in CLF group was 26.92 \pm 3.12 Yrs and in ALF group was 26.90 \pm 3.18 Yrs. Male patients (93.33\%) were more than female patients $(6.67 \%)$. Lachman grading $(\mathrm{mm})$ pre-operative in CLF group was $2.84 \pm 0.35 \mathrm{~mm}$ and in ALF group was $2.72 \pm 0.31$. Maximum patients were from IKDC $2+$ grade. $70.00 \%$ in CLF group and $56.67 \%$ in ALF group. Lysholm score pre-operative in CLF group was 63.00 \pm 1.29 and in ALF group was 62.90 \pm 1.86 . Lachman grading (mm) post-operative in CLF group was $0.31 \pm 0.02 \mathrm{~mm}$ and in ALF group was $0.35 \pm 0$. $06 \mathrm{~mm}$.post-operative $16.67 \%$ patients belong IKDC $2+$ grade in CLF group and in $10.00 \%$ patients in ALF group. Lysholm score post-operative in CLF group was $92.36 \pm 0.95$ and in ALF group was $91.9 \pm 0.91$ There were no statistically significant differences.

\section{Discussion}

Hospital based Prospective Randomized comparative interventional study was conducted at Department of Orthopedics in S.M.S Medical College and Attached Group of hospitals Jaipur, Rajasthan with aims of study was to compare short term outcomes of Arthroscopic ACL reconstruction using fixed closed loop fixation (CLF) and adjustable loop fixation (ALF) devices. In present study, mean age in CLF group was 26.92 \pm 3.12 Yrs and in ALF group was 26.90 \pm 3.18 Yrs. Both groups were comparable. Male patients (93.33\%) were more than female patients $(6.67 \%)$. We used manual Lachman for uniplanner laxity and pivot shift for rotational laxity as the instruments are not available in every clinical setting. To assess knee instability, the most sensitive Lachman test and the most specific pivot shift test is most widely used ${ }^{[8]}$. In present study, Lachman grading $(\mathrm{mm})$ pre-operative in CLF group was $2.84 \pm 0.35 \mathrm{~mm}$ and in ALF group was $2.72 \pm 0.31$. In present study, Maximum patients were from IKDC $2+$ grade. $70.00 \%$ in CLF group and $56.67 \%$ in ALF group. In present study, Lysholm score pre-operative in CLF group was $63.00 \pm 1.29$ and in ALF group was $62.90 \pm 1.86$ There were no significant differences in Lachman and pivot shift grading in CLF and ALF group. There is paucity of clinical published data regarding differentiation of these two fixation techniques. In our experience, the forces required to advance the graft further into tunnel to flip the button were more in closed loop as compared to ALF which allows consistently pull of graft to fixation point. These were created less motion of graft within the tunnel. Some biomechanical studies shows that bone in growth of the graft occurs more readily when there is less motion which leads to less tunnel widening and secure graft fixation ${ }^{[9]}$. An explanation for this is that in CLF, the femoral tunnel required to over drill $0.5 \mathrm{~mm}$ length for clearance of the device through femur cortex and flipping which was not encountered in ALF. We believe that this extra length creates greater motion of graft within the tunnel. This hypothesis remains and requires further prospective clinical data to conclusion. In few biomechanical studies, authors were noted that lengthening and slippage of device of AL lead to knee laxity and delayed graft healing. However, loop of AL device allows retensioning after tibial tunnel fixation ${ }^{[10]}$. We did not experience such outcome in our cases as intraoperatively we have done retensioning of graft after cycling and tibial tunnel fixation. However, there was slight loosening of graft appreciated in all cases of ALF group after application of loading cycles confirmed by anterior drawer, intraoperatively. To overcome these effects, we tied knot over device using pulley sutures in all cases of ALF group to prevent slippage of device. We believe that the advantage is to prevent knee laxity and delayed graft healing after retensioning, but it requires an additional $0.5 \mathrm{~cm}$ incision as disadvantage to push knot inside over device. We have not encountered such slippage in graft with CLF device. In one study, they were not observed slippage of graft. However, there is no mentioning about intraoperative loading cycles and anterior drawer after fixation ${ }^{[11]}$. One biomechanical study evaluates the effects of retensioning and knot tying on the biomechanical properties of an AL device and found that there is an increase in cyclic elongation as compared with CLF during both extended loading conditions which were eliminated by retensioning and knot tying. No clinical study is available on literature search to support knot tying after retensioning of graft to prevent slippage, if encountered after cyclic loading and anterior drawer intraoperatively. Graft rerupture rate was $8.7 \%$ in the CFL and $4.7 \%$ in the ALF group in one study ${ }^{[12]}$. However, results were not statistically significant. We did not find any rerupture of graft on magnetic resonance imaging which was done in those cases showing Grade B laxity by Lachman test 
and Grade 2+ laxity by pivot shift test in both groups, postoperatively. We did not find significant differences in functional outcome using Lysholm score between the groups. Results were consistent with a published study ${ }^{[13]}$. A large sample size and longer follow-up are, therefore, desirable so that we may have more complete set of outcome data to obtain significant differences if any. Inclusion of control group would improve the study.

\section{Conclusion}

We concluded that in femoral fixation of ACL graft both CLF and ALF techniques may provide secure fixation, equal reduction of graft laxity, and similar functional outcome in ACL deficient knee.

\section{Reference}

1. Matthew Frick A, Doris Wenger E, Mark Adkins. MR Imaging of synovial disorders of knee: an update. Radiol Clin N Am 2007;45(6):1017-1031.

2. Anterior cruciate ligament (ACL) injuries - ortho infoAAOS.orthoinfo.aaos.org. March 2014. Archived from the original on 5 July 2017. Retrieved 30 June 2017.

3. Papadakis MA et al., eds. Sports medicine \& outpatient orthopaedics. In: Current Medical Diagnosis \& Treatment 2019. 58 ${ }^{\text {th }}$ ed. New York, N.Y.: McGraw-Hill Education 2019.

4. Polly DW, Callaghan JJ, Sikes RA, McCabe JM, McMahon K, Savory CG. The Accuracy of Selective Magnetic Resonance Imaging Compared with the Findings of Arthroscopy of the Knee. Am J Bone Joint Surg 1988;70:192-198.

5. Vaz CES, Camargo OP de, Santana PJ de, Valezi AC. Accuracy of Magnetic Resonance Imaging in identifying traumatic intraarticular knee lesions. Clinics 2005;60(6):445-50.

6. Hewett TE, Di Stasi SL, Myer GD. Current concepts for injury prevention in athletes after anterior cruciate ligament reconstruction. Am J Sports Med 2013;41:21624.

7. Saccomanno MF, Shin JJ, Mascarenhas R, Haro M, Verma NN, Cole BJ et al. Clinical and functional outcomes after anterior cruciate ligament reconstruction using cortical button fixation versus transfemoral suspensory fixation: A systematic review of randomized controlled trials. Arthroscopy 2014;30:1491-8.

8. Wang C-Y, Wang H-K, Hsu C-Y, Shieh J-Y, Wang T-G, Jiang C-C. Role of sonographic examination in traumatic knee internal derangement. Arch Phys Med Rehabil 2007;88:984-987.

9. Friedman L, Finlay K, Jurriaans E. Ultrasound of the knee. Skeletal Radiol 2001;30:361-377.

10. Hewett TE, Di Stasi SL, Myer GD. Current concepts for injury prevention in athletes after anterior cruciate ligament reconstruction. Am J Sports Med 2013;41:21624.

11. Bosworth DM, Bosworth BM. Use of fascia lata to stabilize the knee in cases of ruptured crucial ligaments. J Bone Joint Surg [Am] 1936;18-A:178-179.

12. Jill E Langer, Susan JF Meyer, Murray K Dalinka. Imaging of the Knee. RadiolClin North Am 1990;28(5):975-990.

13. Rickards D, Chapaman JA. Computed tomography of Anterior Cruciate Ligament. Clinical Radiology 1984;35:327-329. 